\title{
UN NOUVEL ASCARIDE CHEZ RANA ESCULENTA DE PROVENANCE CORSE (1)
}

\author{
Par M. KHALIL
}

Professeur de Parasitologie à la Faculté de médecine du Caire

En disséquant une grenouille, Rana esculenta, provenant de la Corse, le professeur Brumpt a trouvé quatre exemplaires d'un ascaride, l'un dans la portion prépylorique de l'estomac, les autres dans les trois premiers centimètres de l'intestin.

Etant donné la rareté de ces parasites, il me soumit très aimablement ces spécimens pour les déterminer. J'ai constaté qu'ils

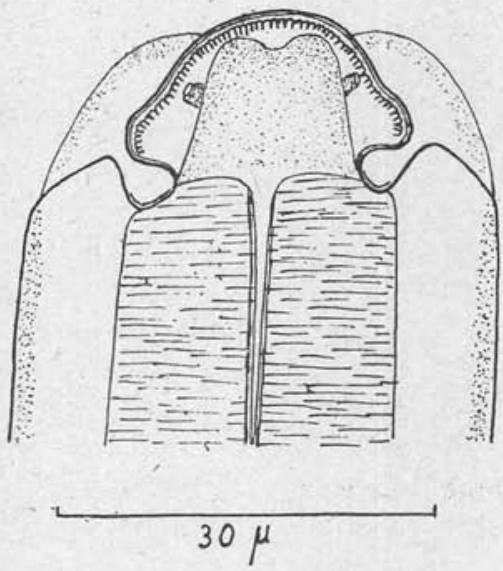

Fig. 1. - Extrémité antérieure d'Amplicœcum brnmpti n. sp., femelle.

appartiennent au genre Amplicocum Baylis, 1920, et qu'ils représentent une espèce nouvelle pour laquelle je propose le nom d'Amplicœcum brumpti n. sp.

Description. - Sur les quatre spécimens examinés, il y avait trois femelles, dont l'une à l'état de maturité sexuèlle et un mâle. Ces vers sont longs et filamenteux, s'enroulant sur eux-mêmes dans le liquide conservateur. La cuticule est épaisse et striée transversalement. L'extrémité postérieure est conique dans les deux sexes

(1) Traduit de l'anglais par M. le Dr H. Galliard.

Annales de Parasitologie, T. IV, $\mathbf{N}^{\circ} 4 .-1^{\text {er }}$ octobre 1926, p. 323-326: 
et plus enroulée chez le mâle que chez la femelle. A l'extrémité antérieure se trouve la bouche, entourée de trois lèvres, l'une dorsale, les deux autres subventrales, de $15 \mu$ à $17 \mu$ de long, munies de crêtes denticulées à la face interne. Il existe des lèvres intermédiaires. La lèvre dorsale porte deux larges papilles, et les deux lèvres subventrales en portent chacune une seule.

L'œsophage est simple, sans bulbe postérieur ; sa longueur est égale au dixième de la longueur totale du corps. Il existe un diverticule intestinal très long de $2 \mathrm{~mm}$., 3 à $2 \mathrm{~mm}$., 7 de long.

Le mâle mesure à peu près $44 \mathrm{~mm}$. de long et $0 \mathrm{~mm}$., 50 de dia-

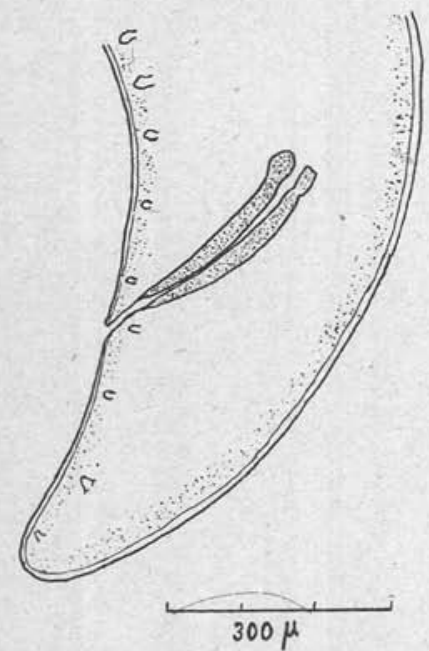

Fıg. 2. - Extrêmité caudale d'Amplicœecum bı umpti n. sp., mâle.

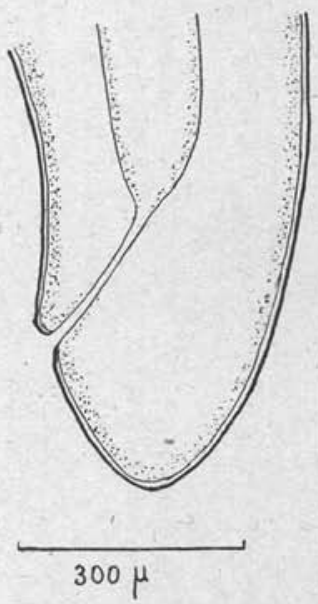

Fig. 3. - Extrémité caudale d'Amplicœeum brumpti n. sp. femelle.

mètre à sa partie la plus épaisse. L'anneau nerveux est situé à $0 \mathrm{~mm} ., 78$ de l'extrémité antérieure. Le diverticule intestinal à 2 mm., 3 de long.

L'extrémité caudale, épaisse et conique, est munie d'ailes bien développées.

Le cloaque est situé à $0 \mathrm{~mm}$., 250 de l'extrémité de la queue. Il y a cinq paires de papilles préanales, trois paires de papilles postanales et en outre une seule papille terminale. Les spicules sont égaux, courts et épais. Ils mesurent $330 \mu$ de longueur. Il n'y a pas de pièce accessoire.

La femelle mesure $70 \mathrm{~mm}$. de long et $0 \mathrm{~mm}$., 75 dans sa partie la plus large. La vulve est située à $19 \mathrm{~mm}$. de la tête, dans le tiers antérieur du corps. L'utérus et les ovaires s'étendent de la vulve 


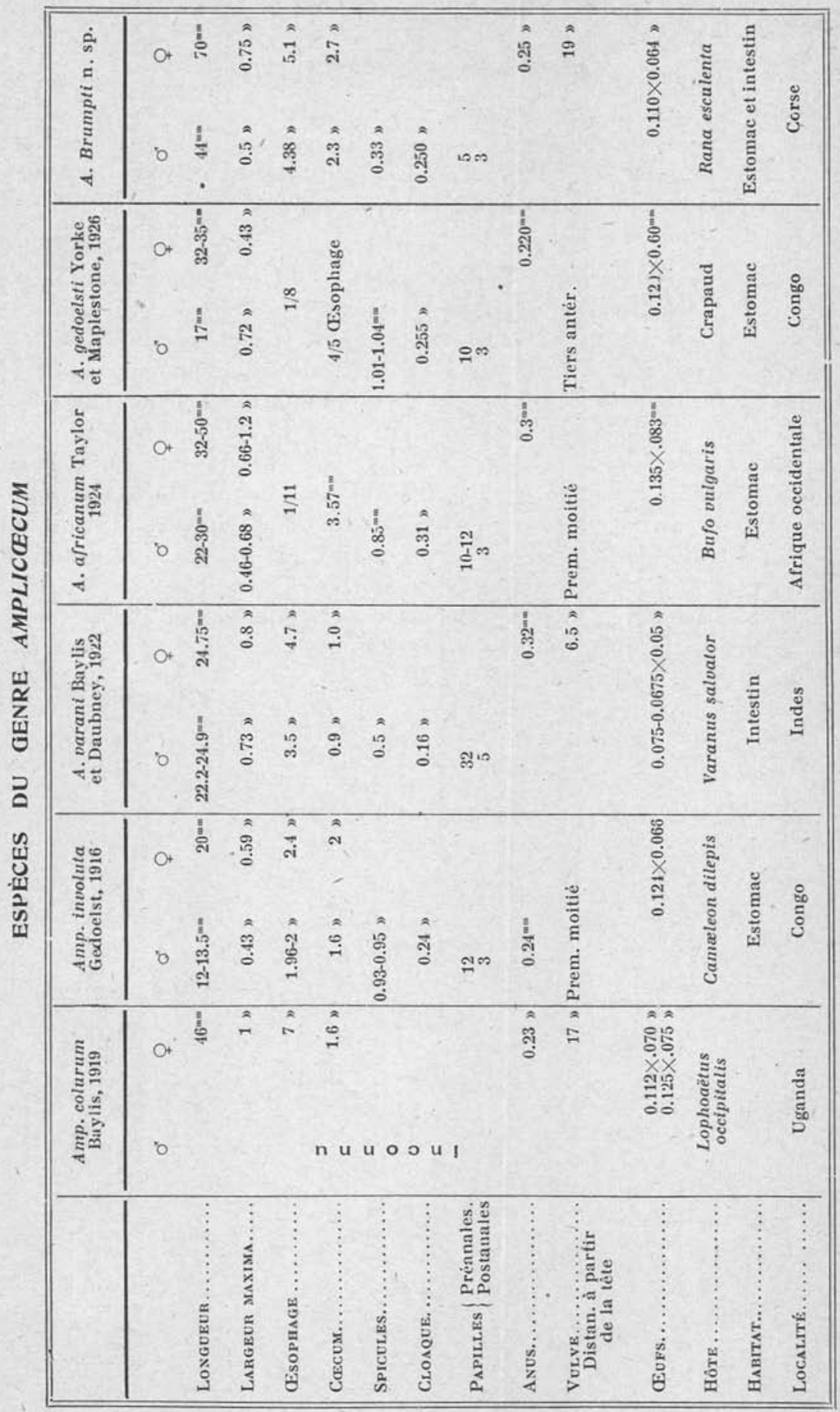


vers la partie postérieure du corps. L'extrémité caudale est large. L'anus est situé à $0 \mathrm{~mm}$., 25 de l'extrémité de la queue. Les œufs sont ovales, entourés d'une coque assez épaisse. Leur surface est finement mamelonnée. Ils mesurent $110 \mu$ de long et $64 \mu$ de large. Ils contiennent un embryon au stade de morula.

Discussion. - Le genre Amplicoecum fut créé par Baylis en 1920, d'après une espèce, $A$. colurum, dont la femelle seule fut décrite; actuellement le genre comprend six espèces'provenant d'hôtes très différents. L'espèce décrite ici est remarquable par ses spicules très courts. Elle est plus longue qu'aucune des autres espèces. Le tableau ci-joint contient les dimensions principales des espèces décrites.

Ces espèces sont surtout caractérisées par la présence de la bordure dentelée de la face interne des lèvres, par un cœecum unique et un œsophage simple. A. africanus possède quelquefois deux cœcums, ce qui prouve que cette structure est sujette à de grandes variations. Les caractères mâles du genre ne peuvent pas être encore strictement définis d'après la description d'un mâle de l'espèce A. colurum. Etant donné les variations énormes de la longueur et de la forme des spicules et des papilles anales, il serait peut-être nécessaire de créer des sous-genres.

\section{BIBLIOGRAPHIE}

Baylis (H.-A.). - Some new Entozoa from birds in Uganda. Ann. and Magaz. of Natural History (9), III, 1919, p. 457.

- On the classification of the Ascaride. Parasitology, XII, 1920, p. 253.

Baylis (H.-A.) et Daubeney (R.). - Parasitic Nematodes in the collection of the Zoological Survey of India. Mem. of the Indian Museum, VII, 1922, p. 263.

Gedoelst (L.). - Notes sur la faune parasitaire du Congo belge. Rev. Zool. africaine, V, 1916, p. 1 .

TAYLOR (E.-L.). - Notes on some Nematodes in the Museum of the Liverpool School of tropical Medicine. Ann. of Trop. Med. and Parasilology, XVIII, 1924 , p. 601.

Yorke (W.) et Maplestone (P.-A.). - The Nematode parasites of vertebrates. London, 1926.

Laboratoire de Parasilologie de la Faculté de médecine de Paris. 